\title{
A multi-aspect assessment for people with mental handicap*
}

An instrument for a systematic assessment of mental handicaps and associated disabilities has been needed for a long time. Recent legislation, for example the Education Act 1981 and the Disabled Person's Act 1986, introduced new statutory responsibilities for education and local authorities to assess an individual's needs. There is no standard assessment or screening tool currently available which covers all aspects of the lives of people with mental handicaps and their interaction with the environment. Ideally a data-base providing such knowledge will also be required by all professions concerned with mental handicap, particularly during the transition to community care.

A multi-professional working party, including coopted members from various disciplines, has been co-ordinated by The Royal College of Psychiatrists.

\section{Objectives}

The main objectives have been:

(1) to establish a common language to define the need(s) of people with mental handicaps

(2) to support individual care plans

(3) to enable the planning of service provision for a defined population

(4) to identify factors which might affect the outcome interventions used in different disabling conditions such as Down's syndrome, autism, etc. in the hope that valid conclusions are drawn from the information available

(5) to enable comparison of different populations (e.g., aetiology, outcome, service provision, resources), etc.

(6) to define a multi-axial structure which might provide a more sensitive classification and for that structure to be compatible with and extend ICD-10

(7) to facilitate clinical audit.

Standard assessment procedures allow valid comparisons to be made between individuals as well as between those in different environments, between different geographical areas and to be monitored over time. People with mental handicaps are a group with diverse disabilities, handicaps and service needs. They vary from those who live virtually independent

Prepared by: Dr Valerie Anness (address for correspondence: Olive Mount Hospital, Old Mill Lane, Liverpool L15 8LW); Mr Ashok Bhat; Dr Nick Bouras; Miss Maria Callias; Professor Sheila Hollins; Mrs Jenifer Rohde; and Professor Ben Sacks (Convenor). lives to those who are better described as multiplihandicapped and a single individual may have psychiatric, physical, CNS, behaviour and other disorders. There are many assessment tools available but none covers all aspects. It is anticipated that the basic structure of the proposed multi-axial format will form the core of different summaries with a varied amount of detail.

The working party has drawn on the work of many other people and looked at assessments in all areas. It has proposed and developed the following seven aspects or axes:

(1) current life context: includes family and living circumstances, service contacts etc.

(2) intellectual: includes level of general learning and reasoning ability

(3) developmental: includes developmental impairments of specific functions such as language, etc.

(4) physical illness including possible causation: i.e. Down's syndrome, epilepsy, etc.

(5) psychiatric and behavioural disorders: includes depression, self injury, etc.

(6) psychosocial factors: includes family relationships, institutional care, etc.

(7) functional adaptation: includes level of overall personal and social functioning in everyday life.

There are three main phases to the work: development, piloting and modification for wider application.

The development work will aim to:

(a) produce the multi-axial system, glossary and instructions

(b) use the system to collect some data

(c) produce some output in the multi-axial format

(d) develop suitable training schemes

(e) consult in detail with all relevant disciplines

(f) modify and refine the system for wider use.

The purpose of the pilot field studies would be:

(a) to assess the operational utility of the system in a wider context in order to ensure its value for different subjects, professions and settings

(b) to ask different professional groups for their assessment of its practicality for their discipline

(c) to modify the forms and output as necessary

(d) the final aim would be the development of a multi-axial classification system in collaboration with WHO for ICD-10. 